\title{
Paroxysmal nocturnal haemoglobinuria and Budd-Chiari syndrome
}

\author{
H A Wyatt, Alex P Mowat, M Layton
}

\begin{abstract}
An 11 year old boy developed pancytopenia, haemolysis, and Budd-Chiari syndrome. The venous thrombosis extended to involve other intra-abdominal vessels before paroxysmal nocturnal haemoglobinuria was recognised as the underlying haematological abnormality. Earlier diagnosis would have made curative bone marrow transplantation a possibility. (Arch Dis Child 1995; 72: 241-242)
\end{abstract}

Keywords: Budd-Chiari syndrome, intravascular haemolysis, hypoplastic anaemia, bone marrow transplantation.

Paroxysmal nocturnal haemoglobinuria (PNH) is a rare but well recognised cause of BuddChiari syndrome. Treatment is most successful if started early in the course of the disease.

\section{Case report}

A boy from Oman presented, at the age of 11 years, with nausea, vomiting and abdominal pain, associated with jaundice but no observed change in urine colour. Over the next 14 months he had two further episodes of jaundice, bruised easily, and became progressively inactive, tired, weak, and thin. Moderate hepatomegaly was the only initial clinical abnormality, but it steadily increased to cause marked abdominal distension with dilated overlying subcutaneous veins that drained cranially, and splenomegaly.

Mild pancytopenia was reported six months after the start of the illness. Further investigation identified a Coombs' negative haemolytic state with a reticulocytosis, hyperbilirubinaemia, and undetectable haptoglobins. Ultrasonographic findings indicated thromboses in the hepatic veins and inferior vena cava.

Eight months later he had developed a profound thrombocytopenia with deteriorating liver function. A surgical procedure to relieve the interior vena caval obstruction was proposed. No local or systemic cause of thrombosis had been identified. A proportionate reduction in multiple procoagulant factors and

King's College

Hospital, Denmark

Hill, London SE5 9RS, Department of Child Health

H A Wyatt

A P Mowat

Department of Haematology

$M$ Layton

Correspondence to Dr Wyatt.

Accepted 30 November 1994 thrombus in the subrenal and intrahepatic portion of the inferior vena cava, splenic vein, and proximal superior mesenteric vein. Surgery could not be recommended with such unexplained, widespread, and apparently progressive thrombosis. The pancytopenia persisted with erythroid hyperplasia on bone marrow examination and continuing haemolysis (table).

The diagnosis of PNH was made by demonstrating increased susceptibility of red blood cells to lysis at an acid pH (Ham's test). ${ }^{1}$ It was confirmed by the reduced expression of CD59, DAF, and LFA3 membrane proteins (molecules that regulate the activation of complement) on almost $100 \%$ of red cells and $20 \%$ of lymphocytes. ${ }^{2}$

\section{Discussion}

The Budd-Chiari syndrome results from obstruction of hepatic venous outflow. Acute occlusion presents with fulminant liver failure. When the onset is more insidious the features are those of cirrhosis, commonly complicated by portal hypertension and gastrointestinal haemorrhage. Causes include vascular compression and endothelial abnormalities as well as prothrombotic defects within the coagulation cascade. ${ }^{3}$

PNH is characterised classically by episodes of intravascular haemolysis occurring during sleep and evidenced by passing red urine on rising. However, less than $25 \%$ of cases have macroscopic haemoglobinuria and haemolysis can occur at any time. Pancytopenia may be the initial feature. Only $12 \%$ of cases occur in patients under 21 years of age and the diagnosis is often delayed in children. ${ }^{4}$

PNH is an acquired disorder, believed to derive from a haemotopoietic stem cell mutation, giving rise to a clone of abnormal blood cells. PNH has recently been shown to be due to a somatic mutation of the phosphatidylinositol glycan class A (PIGA) gene

Secondary haematological abnormalities

\begin{tabular}{lcl}
\hline & $\begin{array}{l}\text { Case } \\
\text { described }\end{array}$ & $\begin{array}{l}\text { Control } \\
\text { normal }\end{array}$ \\
\hline Haemoglobin (g/l) & 86 & \\
Platelet count $\left(\times 10^{9} / \mathrm{l}\right)$ & $9 \cdot 0$ & \\
APTT (sec) & $45 \cdot 5$ & $35 \cdot 0$ \\
Prothrombin time (sec) & $17 \cdot 5$ & $13 \cdot 0$ \\
Factor X (IU//) & $42 \cdot 0$ & $50-150$ \\
Factor VII (IU/1) & $43 \cdot 0$ & $50-150$ \\
Heparin cofactor II (IU/1) & $41 \cdot 0$ & $68-150$ \\
Protein C (IU/) & $39 \cdot 0$ & $70-120$ \\
Plasminogen (IU//) & $65 \cdot 0$ & $75-130$ \\
Postvenous occlusion test: & 127 & \\
$\quad$ Plasminogen activity (\%) & 80 & \\
Antithrombin III activity (\%) & & \\
\hline
\end{tabular}

APTT $=$ activated partial thromboplastin time. the hepatic veins and left branch of the portal vein, with inferior vena caval occlusion secondary to hypertrophy of the uninvolved caudate lobe. Magnetic resonance imaging confirmed these findings and also identified paired hepatic synthesis.

Before surgery he was referred to King's College Hospital for further evaluation. 
on the $\mathrm{X}$ chromosome, which is involved in biosynthesis of the glycosyl phosphatidylinositol (GPI) anchor, to which many cell surface proteins are attached. ${ }^{5}$ Deficiencies of regulatory proteins in the cell membrane render erythrocytes exceptionally sensitive to the lytic effect of complement, encourage aggregation of platelets, and induce relative unresponsiveness of the PNH granulocyte to chemotactic stimuli. $\mathrm{PNH}$ is generally a slowly progressive disease that ends fatally after a few months or many years. Intravascular thrombosis is a well recognised complication with involvement of hepatic, portal, and intracranial veins accounting for significant morbidity and at least $50 \%$ of deaths. Infection or haemorrhage secondary to pancytopenia are the other main causes of mortality.

Conservative treatment aims to minimise the complications of $\mathrm{PNH}$ but is of limited efficacy. Anticoagulation, when thrombosis has been demonstrated, is controversial as secondary haemorrhage may be a greater and even fatal problem. The use of newer thrombolytic agents, for example tissue plasminogen activator, are currently under evaluation. $\mathrm{PNH}$ can be cured by bone marrow transplantation, however, and this is most successful if undertaken before thrombosis is established. ${ }^{6}$

The progression of disease in this case posed a particularly difficult management problem. Any surgical measure would have been danger- ous with such severe thrombocytopenia and may have provoked more extensive thrombosis. Liver transplantation was further contraindicated by the presence of a potentially fatal generalised disease. ${ }^{7}$ Bone marrow transplantation was considered hazardous in the presence of liver disease complicated by portal vein thrombosis. Therefore only supportive management was recommended.

PNH should be suspected in any patient with hypoplastic anaemia or intravascular haemolysis. The presence of venous thrombosis makes the diagnosis highly likely but demands rapid intervention before the opportunity for curative treatment is lost.

Thanks to Peter Hillman, department of haematology, Roya Postgraduate Medical School, Hammersmith Hospital, for the flow cytometric analysis of surface membrane proteins.

1 Grenier-Hughes K. Hemolytic anemias:intracorpuscular defects. In: Pittiglio D Harmening, Sacher RA, eds. Clinical hematology and fundamentals of hemostasis. Philadelphia: FA Davis, 1987: 137-44.

2 Rosse WF. Phosphatidyl-linked proteins and paroxysmal nocturnal hemoglobinuria. Blood 1990; 75: 1595-601.

3 Mowat AP. Liver disorders in childhood. 3rd Ed. Oxford: Mowat AP. Liver disorders in childhood.

4 Ware RE, Hall SE, Rosse WF. Paroxsysmal nocturnal haemoglobinuria with onset in childhood and adolescence. N Engl f Med 1991; 325: 991-6.

5 Takeda J, Miyata T, Kawagoe K, et al. Deficiency of the GPI anchor caused by a somatic mutation of the PIG-A gene in paroxysmal nocturnal haemoglobinuria. Cell 1993; 73: 703-11.

6 Kawahara K, Witherspoon RP, Storb R. Marrow transplantation for paroxysmal nocturnal hemoglobinuria. Am Hematol 1992; 39: 283-8.

7 Calne RY. Contraindications to liver transplantation. Hepatology 1994; 20: 35-45. 